\title{
A NEW ANTI-ALIASING ALGORITHM FOR COMPUTER GRAPHICS IMAGES*
}

\author{
Yuan-Hau Yeh Chen-Yi Lee \\ Dept. of Electronics Engineering, National Chiao Tung University, \\ 1001, University Road, Hsinchu 300, Taiwan, ROC \\ Tel: 886-3-5731849; Email: cylee@cc.nctu.edu.tw
}

\begin{abstract}
This paper presents an area-filtering algorithm for antialiasing technique of computer graphics images. It can be applied to low-resolution image, which the aliasing effect is more visible. Unlike supersampling technique, which costs too much to do well, this technique is simple and suitable for VLSI implementation. Without changing the current PC-based graphics-hardware architecture, this technique costs little overhead and improves the image quality of aliasing images $5 \sim 10 \mathrm{~dB}$ on the average.
\end{abstract}

\section{INTRODUCTION}

Acceleration hardware of real-time 3D graphics has become mainstream in consumer products. Once available primarily on high-end workstations, 3D rasterization hardware has been available on the PC platform since 1995. Due to efforts made by several graphics chip vendors, both performance and quality are greatly improved than the initial products, and within the range of workstation 3D graphics.

Anti-aliasing is an important feature of 3D graphics. There are two popular methods [1] adopted to fulfill this goal, prefiltering and postfiltering. One of the prefiltering algorithm, Catmull's algorithm [2], is an example of an analytic (and expensive) approach using unweighted area sampling. Carpenter's A-buffer algorithm [3] approximates Catmull's algorithm by bit mask and reduces the implementation cost, but the polygons should be in depth-sorted order, which contains complex operations and is not regular for dedicated hardware

\footnotetext{
" WORK SUPPORTED BY NATIONAL SCIENCE COUNCIL OF TAIWAN, R.O.C. UNDER GRANT NSC 88-2215-E-009-068.
}

design. The postfiltering method often uses supersampling of a rendered image, the samples are combined according to a filter weighting in a discrete version of the continuous convolution and sampling. Usually, in order to get ideal image quality, an image large than original image by $4 \times 4$ is created and is down-sampled to original size. For example, the hardware rasterization capacity should reach 960 1280 Mpixels/s (at 400MHz P-II, about $60-80$ Mpixels/sec required for CPU-limited WinBench3D98 score at $800 \times 600$ resolution [4] ) to render $3200 \times 2400$ temporal image. This is far beyond the capacity of existing PC-platform 3D graphics accelerators. And large memory size is demanded to buffer temporal image makes resulting in implementation complexity impossible to be implemented. In this paper, we would like to present one postfiltering method, which is called area filtering, to enhance the aliasing images due to low sampling rate, while the rasterization complexity and memory size are the same as existing $3 \mathrm{D}$ graphics accelerators. The algorithm is discussed in the following sections.

\section{AREA FILTERING ALGORITHM}

The polygons drawn so far have a common problem: they have jagged edges. This undesirable effect is specially visible at high-intensity-transition regions. To eliminate the aliasing effect, we propose a system block diagram as shown in Fig. 1. First, The images generated from rendering hardware are transformed to gray-level images, because aliasing effect is more apparent in high intensity-transition regions. Then Sobel or Prewitt operators are applied to gray-level images and edge bitmaps are generated. From edge bitmaps we can apply 
full search block matching algorithm (BMA) to find block's matched vector (this vector contains edge orientation information). And from matched vectors we know how to filter the aliasing edges by applying the weighting model. In the following sub-sections we will describe each functional block in detail.

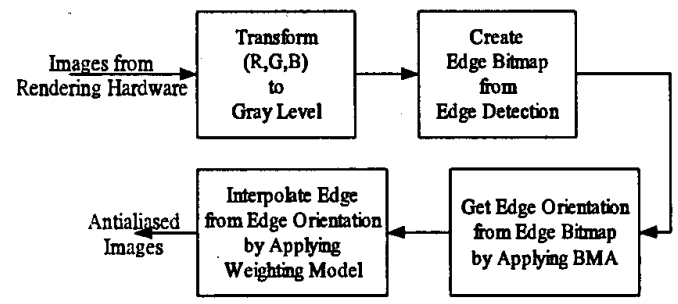

Fig. 1: shows the main functional block within area filtering algorithm.

\subsection{Create Edge Bitmap}

Edge detection has been found to be important as the first step in recognition of objects in images. For objects rendered in computer graphics, the surfaces of each object are relatively invariant to change in luminance. And the aliasing effect usually happens at object (or polygon) junctions. To eliminate the undesired effect, in first step, the luminance information is extracted from rendered color images by converting to YUV format. In the second step, by applying Sobel or Prewitt operators to gray-level images and making the threshold decision, we can get the edge bitmap shown in Fig. 2(a).

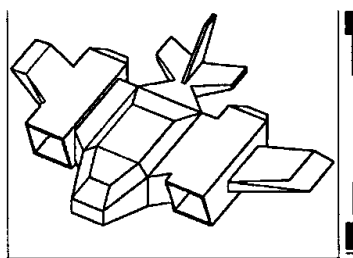

(a)

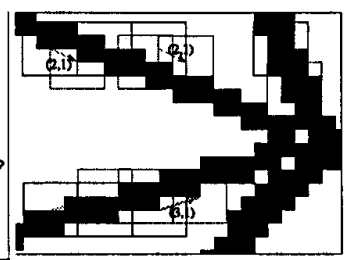

(b)
Fig. 2: (a) edge bitmap. (b) each block which contains edges can find it's matched vector within search range.

\subsection{Find Edge Orientation}

Before filtering aliasing edges, the edge's orientation should be determined. The full search block matching algorithm is a good method to effectively determine edge orientation from the matched vector as shown in Fig. 2(b).
First, image is divided to many blocks, each block contains $N x N$ pixels. In this example, the image is divided to $4 \times 4$ blocks. Because the image is processed in raster scan manner, so for each block, matched vector finder (MVF) only searches bottom half of the search range. From Fig. 2(b) we know the edge usually spans many blocks. These blocks have similar matched vectors, this feature is useful for the weighting model described lately. The advantage of this method is to eliminate most blocks that contain no edges by the simple test, and these blocks need no further process. Table 1 shows that only small part of image contains edges, which needs to be processed.

Table 1: shows only small part of image needs further processing.

\begin{tabular}{|l|c|c|c|}
\hline \multicolumn{1}{|c|}{ Image } & $\begin{array}{c}\text { Total block } \\
\text { count }\end{array}$ & $\begin{array}{c}\text { Block count } \\
\text { (edge) }\end{array}$ & $\begin{array}{c}\text { Percentage } \\
\text { (\%) }\end{array}$ \\
\hline Ship(640x480) & 11844 & 979 & 8.27 \\
\hline Text(320x240) & 2852 & 484 & 17.0 \\
\hline Geometry(640x480) & 11844 & 589 & 4.97 \\
\hline Chair(320x240) & 2852 & 407 & 14.3 \\
\hline
\end{tabular}

\subsection{Establish Weighting Model}

In computer graphics, jagged edges are the result of an all-or-nothing approach to scan conversion in which each is replaced with the polygon's color or is left unchanged. In fact, each polygon contributes color to single pixel depending on it's area covering this pixel. We propose the weighting model to simulate the area covered by polygons along edges. As shown in Fig. 3(a), we classify the edge's orientation into four regions as shown below:

$$
\begin{aligned}
& \text { regionI } m v x \geq m v y . \\
& \text { regionII } m v x<m v y . \\
& \text { regionIII } a b s(m v x)<m v y . \\
& \text { regionIV } a b s(m v x) \geq m v y .
\end{aligned}
$$

While $(m v x, m v y)$ is the matched vector. We only create the weighting model for region I, and this model can be applied to region II IV simply after index substitution as shown in Fig. 3(b). 
The edge can be modeled as two parameters, $\mathrm{M}$ and $\mathrm{N}$. Like stairs, each horizontal bar with size $\mathrm{N}$ stacks another bar with offset $M$, and can be formulated as:

$$
\left\{M=\left\lfloor\frac{\max \{a b s(m v x), m v y\}}{\min \{a b s(m v x), m v y\}}\right\rfloor\right\}
$$

Moving block $\mathrm{i}$ along $+\mathrm{X}$ direction, we can get various block patterns. For each block which contains the edge, we can find corresponding pattern. Each "black" pixel in one block can be assigned one weighting value. As shown in Fig. 4.

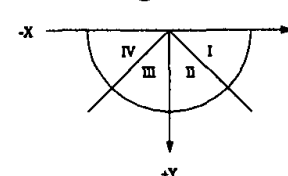

(a)

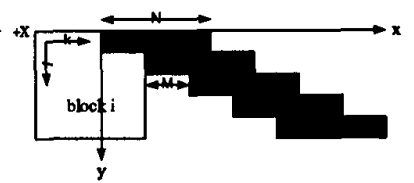

(b)
Fig. 3: (a) edge orientation can be classified into four regions. (b) weighting model.

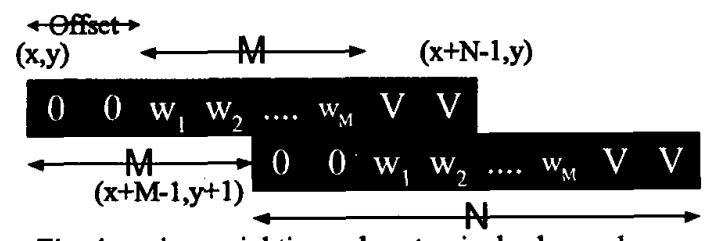

Fig. 4: assign weighting values to pixels along edge.

Each horizontal bar contains $\mathrm{N}$ pixels, and can be divided to three segments:

Segment I: $(\mathrm{x}, \mathrm{y}) \sim(\mathrm{x}+\mathrm{os}-1, \mathrm{y}), \mathrm{w}=0$;

Segment II: $(x+o s, y) \sim(x+o s+M-1, y), w=w_{i}, w_{j}=2 i+1$;

Segment III: $(x+o s+M, y) \sim(x+N-1, y), w=V=2 M$;

While os is the offset value and is equal to (N-M)/2. Finally, each pixel along the edge is blended as:

$C_{\text {pixel }(X, Y)}=(V-w) / V^{*} C_{\text {pixel }(X, Y+1)}+w / V^{*} C_{\text {pixel }(X, Y-1)}$.

\subsection{False Edge Test}

If one block contains multiple edges (comers, vertex shared by multiple polygons or inaccurate edge detection), the block pattern maybe differs from the pattern derived from matched vector largely. So, the different value must be limited to threshold value $T_{D}$ :

$$
\sum_{l=1}^{N} \sum_{k=1}^{N} a b s\left[B_{o}(l, k)-B_{D}(l, k)\right] \leq T_{D}
$$

Where $B_{O}$ is original block pattern, $B_{D}$ is derived block pattern, and $N x N$ is block size. Table 2 shows different threshold value affects image quality. From simulation we know, if we set block size to $5 \times 5$, threshold value is set to 2 or 3 resulting in better PSNR value.

Table 2: different threshold value affects image quality, where block size is $5 \times 5$.

\begin{tabular}{|c|c|c|c|}
\hline \multirow{2}{*}{$T_{D}$} & \multicolumn{3}{|c|}{ PSNR (dB) } \\
\cline { 2 - 4 } & Text & Ship & Geometry \\
\hline 1 & 71.29 & 85.63 & 85.75 \\
\hline 2 & 71.47 & 86.38 & 86.03 \\
\hline 3 & 71.70 & 86.58 & 86.17 \\
\hline 4 & 71.31 & 86.47 & 85.77 \\
\hline 5 & 70.96 & 86.48 & 85.61 \\
\hline
\end{tabular}

\subsection{Continuity Test}

The orientation vectors should not change their direction abruptly along the edges [5]. So, it's necessary to maintain coincident feature of these matched vectors. In Fig. 5, The matched vector of $B(i, j)$ is compared with four processed neighboring blocks and the nearest matched vector $\mathrm{MV}_{\text {nearest }}(\mathrm{x}, \mathrm{y})$ is chosen. If the difference is large beyond the threshold value $T_{C}$, the matched vector is set to $\mathrm{MV}_{\text {nearest }}(\mathrm{x}, \mathrm{y})$.

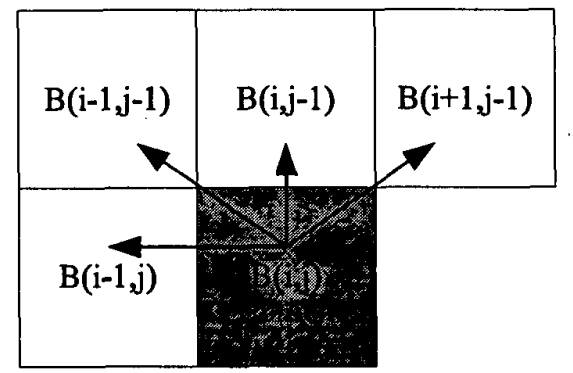

Fig. 5: compare matched vector with four neighboring blocks for continuity test.

\section{RESULTS AND DISCUSSION}




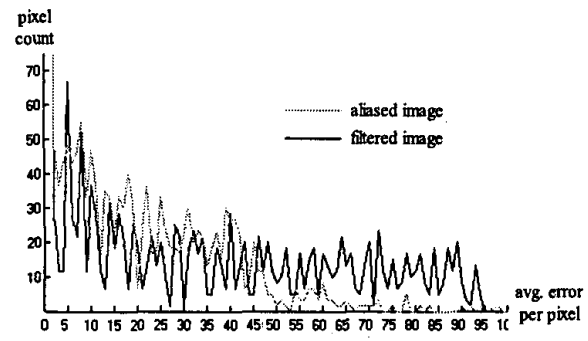

Fig. 6: Error distribution comparison between aliased and filtered "Geometry" images.

We compare aliased and filtered images with $4 \times 4$ supersampling image and find their error distribution as shown in Fig. 6 and Fig. 7. We know that after applying area filtering algorithm, the high error distribution region (i.e. error $>50$ ) is suppressed greatly and moved to low error distribution region caused by approximated error. From test images we know aliasing effect is more visible in high error distribution region. Table 3 shows the PSNR comparison between aliasing and filtering images.

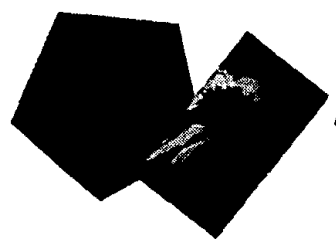

(a)

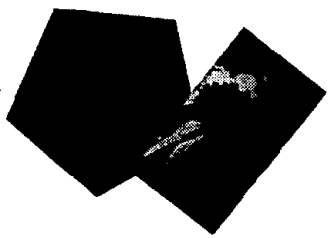

(b)

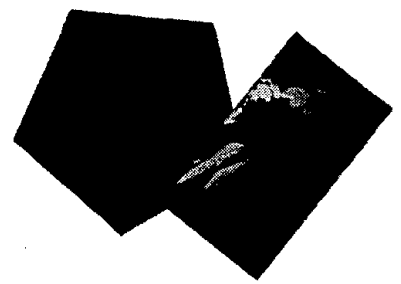

(c)

Fig. 7: (a) 4x4 supersampling "Geometry" image. (b) aliasing image. (c) area filtering image.

Table 3: Image quality comparison in PSNR.

\begin{tabular}{|c|c|c|}
\hline Image & Aliasing image & Filtering image \\
\hline Geometry $(640 \times 480)$ & $81.3 \mathrm{~dB}$ & $86.1 \mathrm{~dB}$ \\
\hline Triangles $(320 \times 240)$ & $77.9 \mathrm{~dB}$ & $90.5 \mathrm{~dB}$ \\
\hline Font $(640 \times 480)$ & $66.7 \mathrm{~dB}$ & $71.7 \mathrm{~dB}$ \\
\hline Ship $(640 \times 480)$ & $83.0 \mathrm{~dB}$ & $89.3 \mathrm{~dB}$ \\
\hline
\end{tabular}

\begin{tabular}{|l|l|l|}
\hline Phoenix (640x480) & $76.6 \mathrm{~dB}$ & $82.3 \mathrm{~dB}$ \\
\hline
\end{tabular}

In summary, our area filtering algorithm is very regular and well-suited for VLSI implementation. Below we highlight its features:

- Computational complexity is reduced: each edgecontained block searches $(2 \mathrm{P})^{2} / 2$ candidates, and each candidate costs only $\mathrm{N}^{2}$ 1-bit absolute difference and accumulation operations. Usually, in each image, only about 1/10 of blocks contain edges. For $800 \times 60060 \mathrm{~Hz}$ animation, about $(1 / 10) *\left[(2 \mathrm{P})^{2} / 2\right] * \mathrm{~N}^{2} * 800 * 600 * 60 / * \mathrm{~N}^{2}$ 1-bit operations are necessary per second. If the search range $-\mathrm{P} \sim \mathrm{P}-1$ is set to $-10 \sim 9$, under $100 \mathrm{MHz}$ clock rate, only $\sim 6$ bit operations are needed per clock cycle.

(2) High oblique edge detection: If the search range is extended, more high oblique edge can be detected. But the penalty paid is the increase of hardware complexity. In our simulation, we set $\mathrm{N}=5$ and $\mathrm{P}=10$.

(3) Blending function is straightforward: there are four types of edge orientation, but only one weighting model is sufficient to handle. The others can access the block patterns only by changing addressing method. The block matches the nearest block pattern and applies blending function. The complexity is similar to matched vector finder.

\section{CONCLUSION AND FUTURE WORK}

In this paper, we have proposed the area filtering algorithm to eliminate aliasing effect of computer graphics images. The image quality can be improved about 5 10 dB without increasing large overhead. It's impossible to find all edge and maintain coherence of edges by using simple edge detection operator. So, more research on accurate and robust edge locator during rendering phase will be conducted in the future.

\section{REFERENCES}

[1] Foley, et. al., Computer Graphics: Principles and Practice, $2^{\text {nd }}$ edition, Addison-Wesley, Nov. 1993.

[2] Catmull, E., "A Hidden-Surface Algorithm with AntiAliasing, "SIGGRAPH 78, pp.6-11. 
[3] Carpenter, L., "The A-buffer, an Antialiased Hidden Surface Method, “ SIGGRAPH 84, pp.103-108.

[4] David B. Kirk, "Unsolved Problems and Opportunities for High-quality, High-performance 3D Graphics on a PC Platform," Eurographics/Siggraph Graphics Hardware Workshop, August 1998, pp.1113.

[5] P. H. Gregson, "Using angular dispersion of gradient direction for detection edge ribbons," IEEE Trans. Pattern Analysis and Machine Intelligence, vol. 15, pp. 682-696, Jul. 1993. 